\title{
Participation of selected soluble cell adhesion molecules and syndecans in formation and development of endometriosis
}

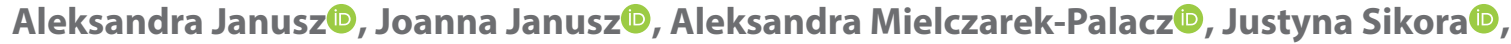

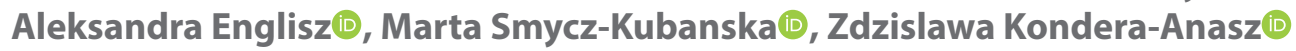 \\ Department of Immunology and Serology, Faculty of Pharmaceutical Sciences in Sosnowiec, \\ Medical University of Silesia, Katowice, Poland
}

\begin{abstract}
Objectives: Concentrations of soluble ICAM-2, $-3,-4$ and syndecan- 1 and -4 have not yet been marked in the peritoneal fluid of women with endometriosis. The aim of the study was to determine whether these molecules can participate in formation and development of endometriosis.

Material and methods: The study comprised of 80 women at the proliferative phase of the menstrual cycle, aged 21 to 49 years (mean age $31.3 \pm 6.7$ years) undergoing laparoscopy, to determine the causes of primary infertility and to confirm or exclude endometriosis. The study group consisted of 60 women with endometriosis in the pelvis as confirmed by laparoscopy and histopathology. The reference group consisted of 20 women in whom no endometriosis. Concentrations of selected sICAM and syndecans in the peritoneal fluid were determined with the use of ELISA method.

Results: Decreased concentrations of SICAM-2 and increased concentrations of SICAM-3, SICAM-4 and syndnecan- 1 and -4 were observed in the peritoneal fluid of women with endometriosis and compared with concentrations of this parameter in the reference group $(p<0.0001)$. Additionally, negative correlation was found between the concentrations of SICAM- 3 and SICAM-2 among women with endometriosis. There was no statistically significant correlation between the concentration of sICAM-2 and SICAM-4, sICAM-3 and SICAM- 4 and syndecan- 1 and syndecan-4 in the examined women.

Conclusions: Changes in concentrations of all the evaluated molecules were observed in the peritoneal fluid in women suffering from endometriosis. Since they have a role in regulation of the immune response, in angiogenesis and apoptosis of the endometrial cells.
\end{abstract}

Key words: endometriosis; soluble intercellular adhesion molecule; sydnecans

Ginekologia Polska 2021; 92, 11: 745-752

\section{INTRODUCTION}

Endometriosis is a chronic disease characterized by the presence of active mucous membrane of the uterus body, beyond its natural location [1,2]. Etiopathogenesis of the disease is still unknown. Still unknown are the mechanisms underlying adhesion of endometrial cells to the peritoneum and enabling their movement to distant regions of the body, initiating development of the ectopic tissue and ensuring its survival [3].

Therefore, among women with endometriosis, it is necessary to conduct research into the role of the molecules responsible for intercellular interactions and the immune response [4-10]. Cell adhesion molecules ICAM (intercellular adhesion molecules) and syndecans participate in migration and adhesion of leukocytes and other body cells and in the process of differentiation and growth of all cells. They are indispensable in maintaining intercellular integrity and are involved in the process of angiogenesis and apoptosis [7, 10]. These molecules form an integral part of the cell membrane of many organs and tissues of the system, but they can also exist in a soluble form in biological fluids. Soluble forms are the result of enzymatic cleavage of extracellular domains of molecules from the cell surface. This can be achieved by means of metalloproteinase-9 (MMP-9, matrix metalloproteinase-9), metalloproteinase-2 (MMP-2, matrix metaloproteinase-2), TNF-a-converting enzyme (TACE, tumor necrosis factor-a converting enzyme) and elastases [11-13]. Soluble adhesion molecules can change activity of appropriate receptors on the cell surface, which can disrupt

Department of Immunology and Serology, Faculty of Pharmaceutical Sciences in Sosnowiec, Medical University of Silesia, Katowice, Poland e-mail: apalacz@sum.edu.pl 
the mechanisms of cell adhesion and migration to remote parts of the system.

No studies have been carried out to assess concentrations of the soluble forms: ICAM-2 (intercellular adhesion molecule-2, CD102), ICAM-3 (intercellular adhesion molecule-3, CD50), ICAM-4, syndecan-1 and 4 in the peritoneal fluid in women with endometriosis.

\section{Aim}

The aim of the study was to determine whether selected soluble cell adhesion molecules and syndecans are involved in formation and development of endometriosis.

\section{MATERIAL AND METHODS}

The study comprised of 80 females aged $21-49$ years (mean age 31.3 \pm 6.7 years) undergoing laparoscopy to determine causes of primary infertility. The examined women were patients of the Department and Clinical Ward of Gynaecology and Obstetrics, Professor. K. Gibiński University Clinical Centre, Medical University of Silesia in Katowice. The inclusion criteria were written, informed consent to participate in the study, regular menstrual cycles lasting $28 \pm 4$ days, overall good health, diagnosis of primary infertility, no history of the use of hormonal agents during the three months before laparoscopy, no history of autoimmune diseases as well as benign or malignant lesions in the ovary and the uterus.

The exclusion criteria were as follows: failure to deliver a written consent to participate in the study, irregular menstrual cycles (not lasting $28 \pm 4$ days), drug history of immunomodulators, hormonal medication, overall poor health, non-malignant or malignant changes in the ovary or in the uterus. 60 women aged $21-49$ years (mean age $31.9 \pm 7.0$ years) were enrolled in the study group, among them endometriosis in the small pelvis was diagnosed during laparoscopy and then confirmed by histopathological examination. The extent and severity of endometrial changes in the peritoneal cavity were evaluated according to the classification of the American Society of Fertility (rAFS), including the division into I, II, III and IV degree of endometriosis. Among 20 women, the first degree of endometriosis was found, among 16 women subjects- II stage, stage III was diagnosed among 15 women, while grade IV was diagnosed among nine women. The reference group consisted of 20 women aged $21-46$ years (mean age $30.4 \pm 6.1$ years) who had no endometriosis or other pathological changes observed in the small pelvic region during laparoscopy. All women were informed about the purpose of the research and they expressed their consent to collection and use of their peritoneal fluid for scientific purposes.

The test material was the peritoneal fluid collected during laparoscopy from women in the proliferative phase of the menstrual cycle. Immediately after collection the peritoneal fluid was centrifuged at $2500 \mathrm{rpm}$. for 10 minutes at $4^{\circ} \mathrm{C}$, in order to separate the fluid from the cells. After centrifugation, the supernatant was divided into small portions and stored at $-80^{\circ} \mathrm{C}$ until the assays were made. The peritoneal fluid concentrations of the following soluble cell adhesion molecules were determined with the use of the Enzyme Linked Immunosorbent Assays (ELISA) by Diaclone SAS, France and Clound-Clone Corp., Houston, USA: sICAM-2 (sensitivity: $0.2 \mathrm{U} / \mathrm{ml}$ ), sICAM-3 (sensitivity: $0.3 \mathrm{ng} / \mathrm{mL}$ ), sICAM-4 (sensitivity: $0.35 \mathrm{ng} / \mathrm{mL}$ ), syndecan-1 (sensitivityt: $4.94 \mathrm{ng} / \mathrm{mL}$ ) and syndecan-4 (sensitivity: $0.125 \mathrm{ng} / \mathrm{mL}$ ).

The obtained results were statistically analysed with the use of Statistica for Windows version 12.0 and MS Excel. The Shapiro-Wilk test was used to assess distribution of the obtained results. In case of the obtained results corresponding to normal distribution, $t$-Student test was used for independent samples to analyse the results. The arithmetic mean ( $\mathrm{x}$ ) and standard deviation (SD) were calculated for each parameter. In the case of the homogeneity principle of variance of the tested parameters, a one-way ANOVA analysis was used. The significance of differences was additionally verified by means of the Rir Tukey post-hoc test. For given features which differed significantly, the nonparametric methods were used to compare the results and the median and interquartile ranges were determined - the lower quartile (Q1) and the upper quartile (Q3). In this case, the Mann-Whitney U test and the Kruskal-Wallis RAN ANOVA test were used. In case of statistically significant differences, a multiple post-hoc test was used. For normal distribution, correlation between the tested parameters was verified using the Pearson's correlation coefficient ( $r$ ). If the assumption of normality of distribution was not met, the Spearman's rank correlation coefficient $(\mathrm{R})$ was calculated. The assumed significance level was $p \leq 0.05$.

\section{Ethical approval}

The approval of the Bioethics Committee, e Medical University of Silesia in Katowice, Poland, No. NN-6501-57/07, of April 4, 2007 was obtained for the tests.

\section{RESULTS}

In the peritoneal fluid of women with endometriosis a decrease in sICAM-2 concentration was found, as compared to the concentration of this parameter in the reference group ( $p<0.0001)$. The mean sICAM-2 concentration among women with endometriosis was $34.13 \pm 16.84$ (3.90-89.98), and in the reference group: $58.26 \pm 8.44$ (37.01-74.80). A statistically significant decrease in SICAM- 2 concentration in peritoneal fluid of women with I, II and IV degree of the disease was demonstrated, as compared to concentrations of this parameter in the reference group $(p<0.0001$ and 


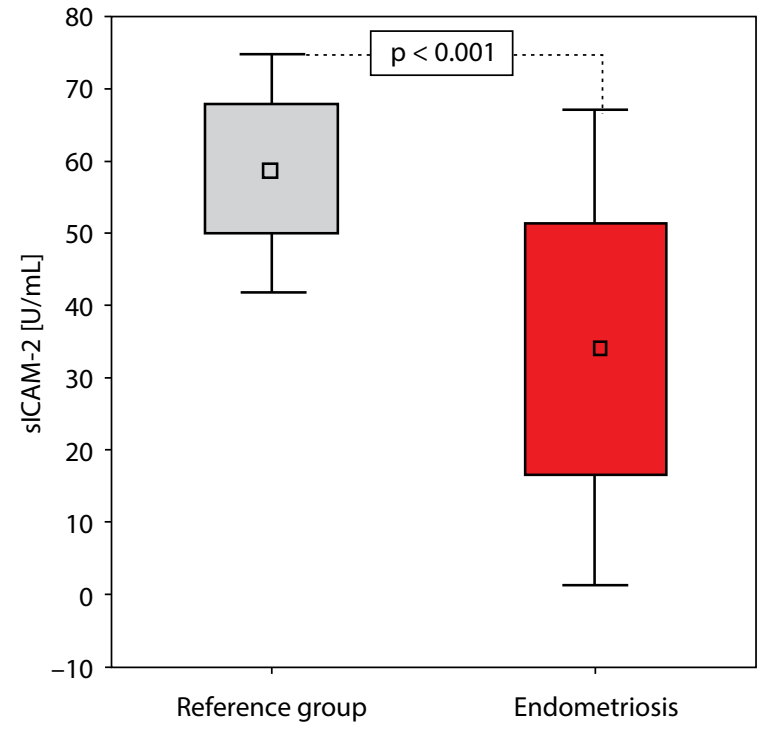

Figure 1. sICAM-2 concentration in the peritoneal fluid of women in the reference group and women with endometriosis

$p<0.0001$ and $p<0.0001$ respectively). In contrast, the concentration of sICAM- 2 in the peritoneal fluid of women with stage III was not statistically reduced, comparing to concentrations of this parameter among females in the reference group. There was no statistically significant correlation between the concentration of SICAM-2 in the peritoneal fluid and the subsequent advancement of endometriosis among the examined women. The results are illustrated in Figure 1 and presented in Table 1.

Elevated concentrations of sICAM-3 were found in the peritoneal fluid of women with endometriosis, comparing to concentrations of this parameter in the reference group ( $p<0.0001)$. The mean sICAM-3 concentration among women with endometriosis was $18.63 \pm 5.18$ (10.87-37.64), comparing to the reference group: $11.86 \pm 3.08$ (5.84-19.95). Analysis of the results showed a statistically significant increase in SICAM-3 concentration in the peritoneal fluid among women with I and IV stage of the disease and statistically significant increase in the concentration of the tested parameter among women with endometriosis II, comparing to SICAM-3 in the reference group (respectively $p<0.0001$ and $p<0.0001$ and $p<0.001)$. On the contrary, concentrations of SICAM-3 in the peritoneal fluid of women with stage III of the disease was not significantly increased comparing to the concentrations of this parameter among women in the reference group. The study showed no statistically significant correlations between the concentration of sICAM-3 in the peritoneal fluid and the successive stages of endometriosis. Negative correlation was found between SICAM-3 and SICAM- 2 concentrations among women with endometriosis. The results are illustrated in Figures 2 and 3 and presented in Table 2.
Table 1. sICAM-2 concentration in the peritoneal fluid of women at subsequent stages of endometriosis and women in the reference group

\begin{tabular}{|c|c|c|c|c|}
\hline \multicolumn{2}{|l|}{ Examined group } & \multirow[b]{3}{*}{20} & \multicolumn{2}{|c|}{$\begin{array}{l}\text { sICAM-2 concentration in the } \\
\text { peritoneal fluid }[\mathrm{U} / \mathrm{mL}]\end{array}$} \\
\hline & & & \multirow{2}{*}{$\begin{array}{l}\text { range } \\
6.27-50.01\end{array}$} & \multirow{2}{*}{$\begin{array}{l}\mathbf{x} \pm \text { SD } \\
30.17 \pm 12.35^{*}\end{array}$} \\
\hline \multirow{4}{*}{$\begin{array}{l}\text { Women at } \\
\text { subsequent stages } \\
\text { of endometriosis }\end{array}$} & 1 & & & \\
\hline & $\|$ & 16 & $5.75-57.19$ & $37.06 \pm 11.41^{*}$ \\
\hline & III & 15 & $27.78-89.98$ & $49.59 \pm 14.73^{* *}$ \\
\hline & IV & 9 & $3.90-24.81$ & $11.97 \pm 6.90^{*}$ \\
\hline \multicolumn{2}{|l|}{$\begin{array}{l}\text { Women from the } \\
\text { reference group }\end{array}$} & 20 & $37.01-74.80$ & $58.26 \pm 8.44$ \\
\hline
\end{tabular}

$\mathrm{n}$ - group size; $\mathrm{x}$ - arithmetical mean; SD — standard deviation; ${ }^{*} p<0.0001$ compared to women in the reference group; ${ }^{* *}$ NS compared to women in the reference group

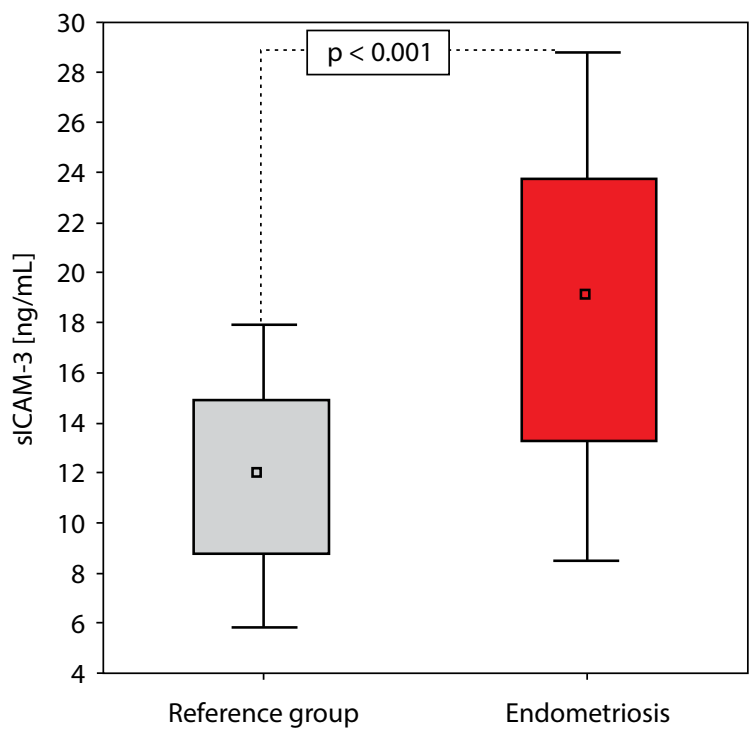

Figure 2. sICAM-3 concentration in the peritoneal fluid of women in the reference group and women with endometriosis

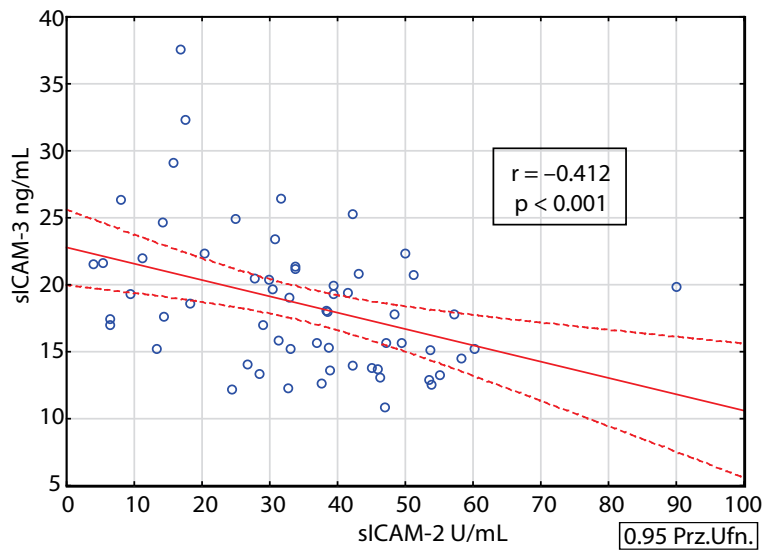

Figure 3. Linear regression curve illustrating the correlation between concentration of SICAM-3 and SICAM- 2 in the peritoneal fluid of women with endometriosis 


\begin{tabular}{|c|c|c|c|c|}
\hline \multirow{2}{*}{\multicolumn{2}{|c|}{ Examined group }} & \multirow[t]{2}{*}{$\mathbf{n}$} & \multicolumn{2}{|c|}{$\begin{array}{l}\text { sICAM-3 concentration in the } \\
\text { peritoneal fluid }[\mathrm{ng} / \mathrm{mL}]\end{array}$} \\
\hline & & & range & $x \pm S D$ \\
\hline \multirow{4}{*}{$\begin{array}{l}\text { Women at } \\
\text { subsequent } \\
\text { stages of } \\
\text { endometriosis }\end{array}$} & 1 & 20 & $12.24-23.43$ & $18.52 \pm 2.84^{*}$ \\
\hline & $\|$ & 16 & $12.27-29.16$ & $17.46 \pm 4.90^{* *}$ \\
\hline & III & 15 & $10.87-26.48$ & $15.97 \pm 4.03^{* * *}$ \\
\hline & IV & 9 & $17.02-37.64$ & $25.36 \pm 6.22^{*}$ \\
\hline \multicolumn{2}{|l|}{$\begin{array}{l}\text { Women in the } \\
\text { reference group }\end{array}$} & 20 & $5.84-19.95$ & $11.86 \pm 3.08$ \\
\hline
\end{tabular}

$\mathrm{n}$ - group size; $\mathrm{x}$ - arithmetical mean; SD — standard deviation;

${ }^{*} p<0.0001$ compared to women in the reference group; ${ }^{* *} p<0.001$ compared to women in the reference group; ${ }^{* * *} \mathrm{NS}$ compared to women in the reference group

An increase in sICAM-4 concentration was found among women with endometriosis, comparing to concentrations of this parameter in the reference group $(p<0.0001)$ and the mean sICAM-4 concentration was 0.89 (Q1: 0.39 and Q3: 1.93 and $0.18-7.57)$, and in the reference group: 0.28 (Q1: 0.13 and Q3: 0.39 and 0.05-0.53). Analysis of the results showed a significant increase in SICAM-4 concentration in the peritoneal fluid among women with stage I and II stage of the disease, as compared to concentrations of this parameter in the fluid of females in the reference group $(p<0.0001$ and $p<0.0001$ respectively). However, the concentration of sICAM-4 in the peritoneal fluid among women with stage III and IV was not statistically increased, comparing to concentrations of this parameter among women in the reference group.

Moreover, a statistically significant negative correlation between SICAM- 4 concentration and successive stages of the disease in the peritoneal fluid of the examined women $(R=-0.633$ and $p<0.0001)$ was demonstrated. There was no statistically significant correlation between the concentration of sICAM- 2 and SICAM-4, sICAM-3 and SICAM-4 among the examined women. The results are illustrated in Figures 4 and 5 and presented in Table 3.

The tests revealed increased peritoneal fluid concentrations of syndecan-1 among women with endometriosis, comparing to the concentrations of this parameter in the reference group $(p<0.0001)$. Mean concentration of syndecan-1 reached 11.42 (Q1: 7.72 and Q3: 15.62 and 2.35-33.20), as compared the reference group: $4.28(\mathrm{Q} 1: 2.80$ and Q3: 5.75 and $0.88-8.50$ ). Analysis of the results showed a statistically significant increase in syndecan- 1 concentrations in the peritoneal fluid among women with stage I and IV, and statistically significant increase in the test parameter among the females with stage II of endometriosis, compared to syndecan- 1 in the reference group $(p<0.0001 p<0.0001$ and $p<0.001$ ). However, concentrations of syndecan-1 in the peritoneal fluid of women with stage III was not statistically

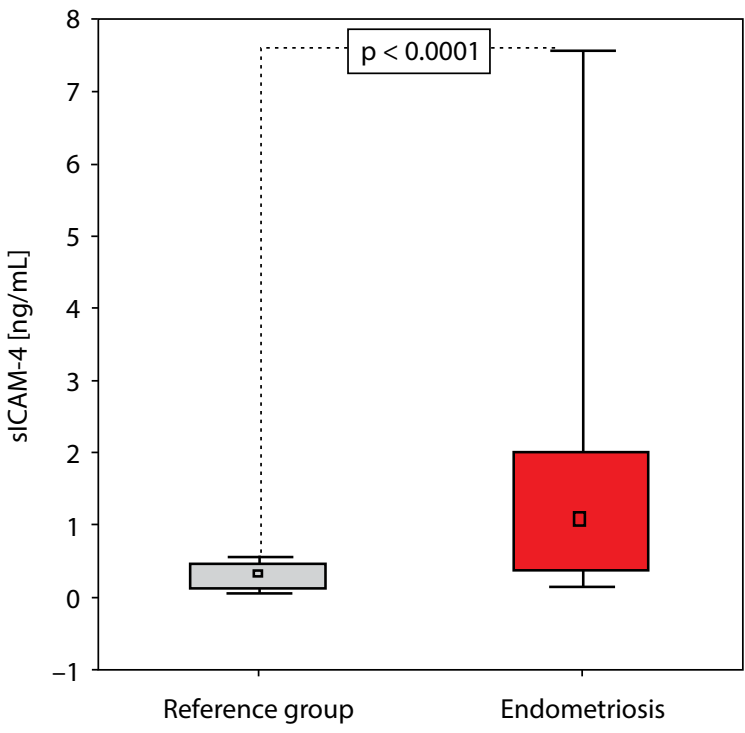

Figure 4. sICAM-4 concentration in the peritoneal fluid of women in the reference group and women with endometriosis

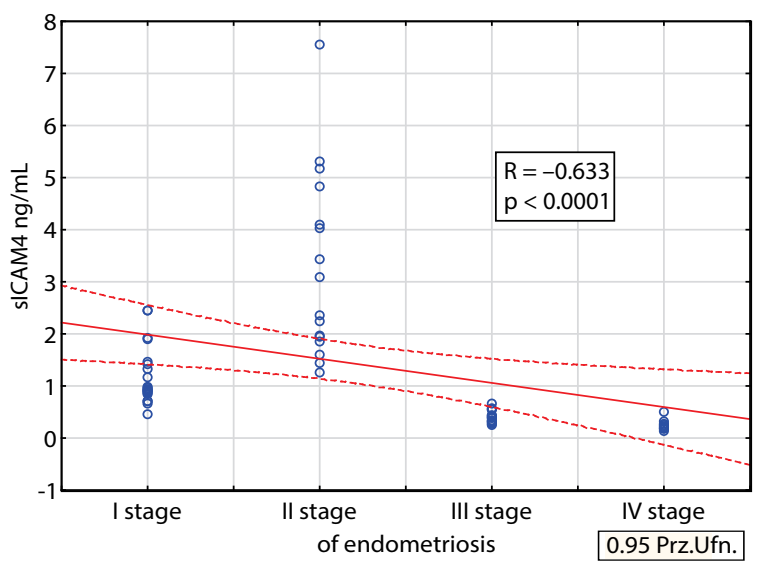

Figure 5. Linear regression curve illustrating the correlation between concentration of SICAM-4 in the peritoneal fluid of women with endometriosis and the subsequent stages of disease

Table 3. sICAM-4 concentration in the peritoneal fluid of women at subsequent stages of endometriosis and women in the reference group

\begin{tabular}{|c|c|c|c|c|c|c|}
\hline \multirow{2}{*}{\multicolumn{2}{|c|}{$\begin{array}{l}\text { Examined } \\
\text { group }\end{array}$}} & \multirow[b]{2}{*}{$n$} & \multicolumn{4}{|c|}{$\begin{array}{l}\text { sICAM- } 4 \text { concentration in the peritoneal } \\
\text { fluid [ng/mL] }\end{array}$} \\
\hline & & & median & range & $\begin{array}{l}\text { Lower } \\
\text { quartile } \\
\text { Q1 }\end{array}$ & $\begin{array}{l}\text { Upper } \\
\text { quartile } \\
\text { Q3 }\end{array}$ \\
\hline \multirow{4}{*}{$\begin{array}{l}\text { Women at } \\
\text { subsequent } \\
\text { stages of } \\
\text { endome- } \\
\text { triosis }\end{array}$} & 1 & 20 & $0.96^{*}$ & $0.47-2.46$ & 0.86 & 1,45 \\
\hline & ॥ & 16 & $2.73^{*}$ & $1.26-7.57$ & 1.91 & 4,50 \\
\hline & III & 15 & $0.39^{* *}$ & $0.26-0.68$ & 0.32 & 0,56 \\
\hline & IV & 9 & $0.23^{* *}$ & $0.14-0.52$ & 0.19 & 0,28 \\
\hline \multicolumn{2}{|c|}{$\begin{array}{l}\text { Women in the } \\
\text { reference group }\end{array}$} & 20 & 0.28 & $0.05-0.53$ & 0.13 & 0.39 \\
\hline
\end{tabular}

$\mathrm{n}$ - group size; ${ }^{*} \mathrm{p}<0.0001$ compared to women in the reference group; ${ }^{* *} \mathrm{NS}$ compared to women in the reference group 




Figure 6. Syndecan-1 concentration in the peritoneal fluid of women in the reference group and women with endometriosis

increased comparing to concentrations of this parameter among women in the reference group. There was no statistical correlation between the peritoneal fluid concentrations of syndecan-1 in further stages of advanced endometriosis among the examined women. The results are illustrated in Figure 6 and presented in Table 4.

Increased peritoneal fluid syndecan-4 concentrations were observed in women with endometriosis, as compared to concentrations of this parameter in the reference group ( $p<0.0001)$. Mean concentration of syndecan- 4 was $16.66 \pm 7.12$ (1.72-42.03), and: $10.05 \pm 3.65$ (3.51-15.3) in the reference group. The statistical analysis showed a significant increase in concentrations of syndecan- 4 in the peritoneal fluid of women with stage I and a significant increase in the test parameter among women with stage II of the disease, as compared to syndecan-4 in the reference group $(<0.0001$ and $p<0.01$ respectively). However, concentrations of syndecan-4 in the fluid of women with stage III and IV was not statistically increased comparing to concentrations of this parameter among women in the reference group. In addition, a significant correlation was found between the concentrations of syndecan- 4 in the peritoneal fluid and further stages of the disease among the examined women $(R=-0.635$ and $p<0.0001)$. There was no statistical correlation between concentrations of syndecan-1 and syndecan-4 in the evaluated females. The results are illustrated in Figures 7 and 8 and shown in Table 5.

\section{DISCUSSION}

The receptors for the ICAM-2 molecule are: LFA-1 integrin on leukocytes and DC-SIGN lectin (Dendritic Cell-Specyfic Intercellular adhesion molecule-3 grabbing Non-integrin) locat-
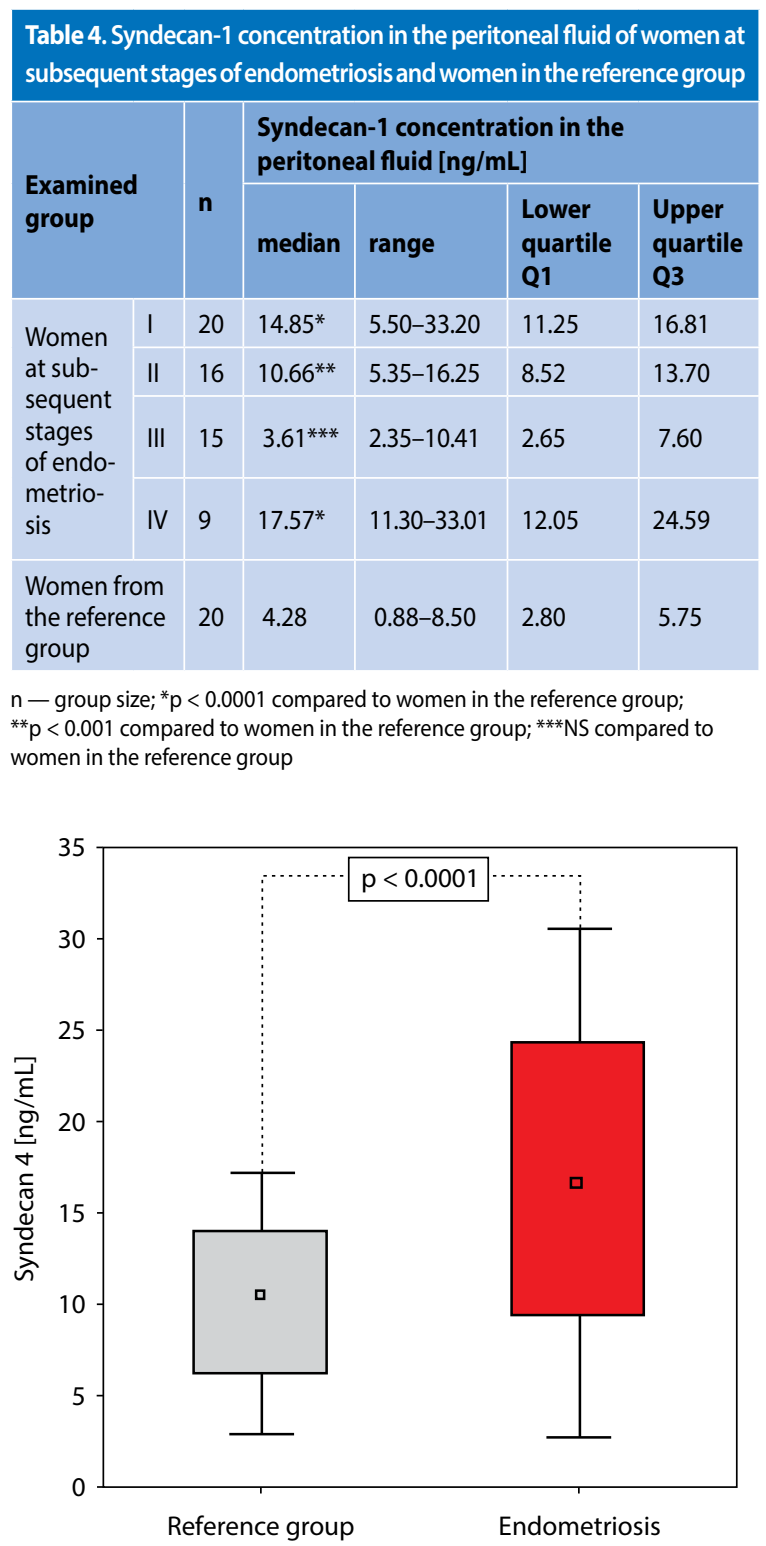

Figure 7. Syndecan-4 concentration in the peritoneal fluid of women in the reference group and women with endometriosis

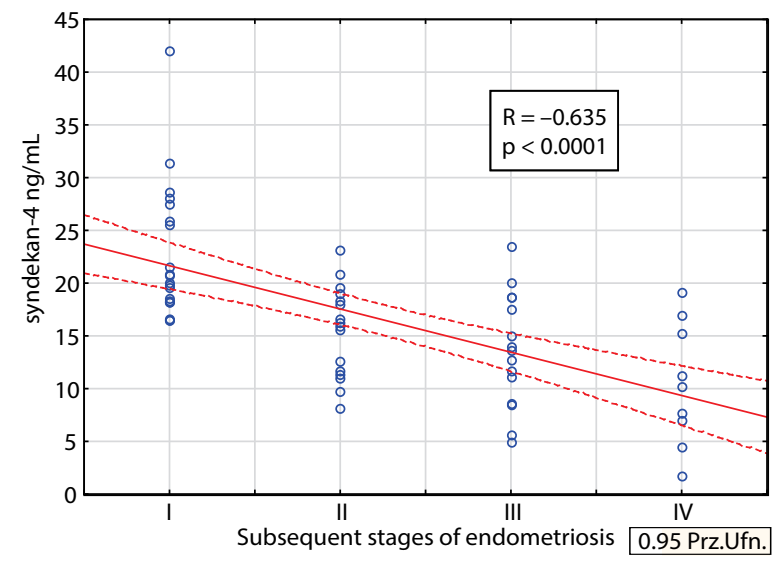

Figure 8. Linear regression curve illustrating the correlation between concentration of syndecan-4 in the peritoneal fluid of women with endometriosis and the subsequent stages of the disease 


\begin{tabular}{|c|c|c|c|c|}
\hline \multirow{2}{*}{\multicolumn{2}{|c|}{ Examined group }} & \multirow[t]{2}{*}{$\mathbf{n}$} & \multicolumn{2}{|c|}{$\begin{array}{l}\text { Syndecan- } 4 \text { concentration in } \\
\text { the peritoneal fluid [ng/mL] }\end{array}$} \\
\hline & & & range & $x \pm S D$ \\
\hline \multirow{4}{*}{$\begin{array}{l}\text { Women atsubsequent } \\
\text { stages of endome- } \\
\text { triosis }\end{array}$} & 1 & 20 & $16.45-42.03$ & $22.71 \pm 6.40^{*}$ \\
\hline & $\|$ & 16 & $8.15-23.16$ & $15.50 \pm 4.32^{* *}$ \\
\hline & III & 15 & $4.92-23.50$ & $13.61 \pm 5.42^{* * *}$ \\
\hline & IV & 9 & $1.72-19.17$ & $10.39 \pm 5.84^{* * *}$ \\
\hline \multicolumn{2}{|l|}{$\begin{array}{l}\text { Women in the reference } \\
\text { group }\end{array}$} & 20 & $3.51-15.31$ & $10.05 \pm 3.65$ \\
\hline
\end{tabular}

$\mathrm{n}$ - group size; $\mathrm{x}$ - arithmetical mean; SD — standard deviation;

$p<0.0001$ compared to women in the reference group; ${ }^{* *} p<0.001$ compared to women in the reference group; ${ }^{* * *} \mathrm{NS}$ compared to women in the reference group

ed on dendritic cells $[4,5,7,9,10,14-17]$. Thus, the activity of lymphocytes and dendritic cells may modulate. Consequently, it may lead to incorrect recognition of the endometrial cells in ectopic sites. Amsellem et al. [11] studied participation of sICAM-2 in the process of cancer cell migration. According to the authors, the soluble ICAM-2 molecule facilitates migrating cells to overcome the vascular endothelium barrier and to move to distant parts of the system.

A soluble ICAM-3 molecule may cause impairment of LFA-1 receptor bonding to other ICAM molecules. As the ICAM-3 molecule is involved in both, antigen presentation and stimulation of lymphocytes, as well as in the effector phase of the immunological response, the increased concentration of the soluble form of this molecule in the peritoneal fluid of women with endometriosis may contribute to impairment of both phases. The DC-SIGN receptor located on dendritic cells, connecting with the ICAM-3 molecule on virgin T lymphocytes, supports the diagnosis of the presented antigen [17]. If sICAM-3 is released, the recognition and presentation of the antigen may be impaired [16]. Combining with the DC-SIGN receptor on NK cells and macrophages and with the LFA-1 receptor on CD8 + T lymphocytes, the soluble sICAM-3 molecule reduces activity of these cells. The effect of such combination may weaken or eliminate the cytotoxic activity directed against the target cells. It has been shown in endometriosis that peritoneal macrophages and NK cells show limited ability of eliminating the endometrial cells from the peritoneal cavity [18].

A negative correlation between the concentration of sICAM-3 and sICAM-2, shown in the study, may prove that these molecules compete for the binding site of the appropriate receptors, both on leukocytes and dendritic cells, as well as on vascular endothelial cells. This may contribute to abnormal adhesion of the immune system to the vascular endothelium and abnormal leukocyte migration into the sites where the ectopic cells of the endometrium were im- planted. This may result in abnormal immune response to the ectopic endometrium cells [18-20].

Analysis of the results showed increased concentration of sICAM-4 in the peritoneal fluid of women with endometriosis, comparing to concentrations of this parameter in the reference group. This particularly concerned women with stage I and II of the disease. It may point to participation of sICAM-4, especially at the early stage of the disease. Lacking outflow, in endometriosis the menstrual red blood cells undergo haemolysis. It leads to the unveiling of phosphatidylserine and, due to the lack of its outflow, intensified expression of ICAM-4 molecule on red blood cells and the release of its soluble form. This contributes to the increased binding capacity of sICAM- 4 on erythrocytes, integrin receptors located on vascular endothelium, monocytes, leukocytes, platelets and NK cells and it promoting the risk of adhesion of other cells to its surface, perhaps also the ectopic endometrium cells.

Negative correlation between SICAM- 4 concentrations in the peritoneal fluid of women with endometriosis and the subsequent stages of the disease was also demonstrated. It is possible that the increased concentration of sICAM- 4 in the peritoneal fluid of women with endometriosis is due to the presence of active ectopic endometrium cells and indicative of the early stage of the disease. In contrast, the decrease in SICAM-4 concentration found in the peritoneal fluid of women with endometriosis may suggest the presence of inactive and fibrotic endometriotic changes, observed in women with advanced endometriosis.

The soluble syndecan- 1 retains the activity of the membrane form. For this reason, the molecule can compete with its counterpart on the surface of the cell, for the binding site of the appropriate receptor and modulate the biological functions of the cell. Binding soluble syndecan-1 to growth factors allows for cell proliferation. The presence of soluble syndecan-1 in the peritoneal fluid may therefore facilitate survival of the endometrial cells outside the uterine cavity.

The expression of syndecan-1 has been demonstrated on endometrial cells. Schneider et al. [21], demonstrated participation of syndecan-1 in proliferation and invasiveness of the endometrial cells and in development of inflammation in women with endometriosis. The authors believe that syndecan-1 allows for implantation of these cells in the ectopic sites. According to the authors, the increase of cell invasiveness is because of syndecan- 1 on secretion of interleukin- 6 (interleucine-6) and modulation of the proteolysis process. Increased syndecan-1 concentrations in the peritoneal fluid, observed during the study in women with endometriosis, may point to participation of this molecule in the processes mentioned above. Relationship between the increased expression of the membranous form of syn- 
decan-1 on the endometrial cells and formation of endometriosis was indicated by Cicinelli et al. [22].

Changes in concentrations of soluble syndecan-4 observed in the study in women with endometriosis may result in increased proliferation of the smooth muscle cells in the blood vessels and in formation of their basement membrane [22]. Formation of new vessels is necessary for survival of the ectopic endometrium implants outside the uterine cavity. The syndecan- 4 molecule plays an important role in repair of the damaged tissues. During skin regeneration, expression of syndecan-4 is increased in the granulation tissue on the endothelial cells and fibroblasts [23]. At stage IV endometriosis, the ectopic endometrium becomes fibrotic. Chelariu-Raicu et al. [6] showed that syndecan-4 might participate in pathogenesis of endometriosis through the influence of this molecule on metalloproteinase-3, GTP-azaR1 and ATF-2 (activating transcription factor-2.) These researchers showed that syndecan-4 contributed to the increased endometrial cell activity, allowing for their development in the ectopic sites, whereas Schneider et al. [20] showed that syndecan-4 inhibited proliferation of these cells by reducing metalloproteinase- 9 activity and interleukin- 6 concentration. (IL-6).

In conclusion, changes in concentrations of all the soluble molecules evaluated during the study were demonstrated in the peritoneal fluid of women with endometriosis.

\section{Key message}

Syndecans and ICAMs are involved in regulation of the immune response and as a result of such changes, there may occur an abnormal response directed against the ectopic endometrium cells, facilitating their transfer and implantation in distant sites.

\section{CONCLUSIONS}

The conducted research led to the following conclusions:

1. Changes in the concentration of the tested soluble cell adhesion molecules and syndecans, especially visible in the first and second degree of the disease, may indicate the participation of these molecules in the formation of endometriosis and indicate the intensification of migration and adhesion processes of endometrial cells in these stages of the disease.

2. Observed changes in the concentration of the tested soluble molecules, found in the peritoneal fluid of women with advanced endometriosis, may indicate the participation of these molecules in the development of the disease, by affecting the adhesion of endometrial cells to vascular endothelial cells and peritoneal mesothelium and migration to distant places of the body.
3. The assessed molecules, by participating in repair processes, such as fibrosis and scarring, may contribute to the deformation of organs affected by the disease process and may allow the formation of blood vessels in the tissue of the ectopic endometrium, which allows these cells to survive outside the uterine cavity.

\section{Conflict of interest}

No conflict of interest was declared by the authors.

\section{REFERENCES}

1. Bohonyi N, Pohóczky K, Szalontai B, et al. Local upregulation of transient receptor potential ankyrin 1 and transient receptor potential vanilloid 1 ion channels in rectosigmoid deep infiltrating endometriosis. Mol Pain. 2017; 13: 1744806917705564, doi: 10.1177/1744806917705564, indexed in Pubmed: 28478727.

2. Galvankar M, Singh N, Modi D. Estrogen is essential but not sufficient to induce endometriosis. J Biosci. 2017; 42(2): 251-263, doi: 10.1007/s12038-017-9687-4, indexed in Pubmed: 28569249.

3. Johnson NP, Hummelshoj L, Adamson GD, et al. World Endometriosis Society Sao Paulo Consortium . World Endometriosis Society consensus on the classification of endometriosis. Hum Reprod. 2017; 32(2): 315-324, doi: 10.1093/humrep/dew293, indexed in Pubmed: 27920089.

4. Bessa NZ, Francisco Dd, Andres Md, et al. Polymorphisms of ICAM-1 and IL-6 genes related to endometriosis in a sample of Brazilian women. J Assist Reprod Genet. 2016; 33(11): 1487-1492, doi: 10.1007/s10815016-0796-z, indexed in Pubmed: 27614631.

5. Carulli S, Beck K, Dayan G, et al. Cell surface proteoglycans syndecan-1 and -4 bind overlapping but distinct sites in laminin a3 LG45 protein domain. J Biol Chem. 2012; 287(15): 12204-12216, doi: 10.1074/jbc. M111.300061, indexed in Pubmed: 22351752.

6. Chelariu-Raicu A, Wilke C, Brand M, et al. Syndecan-4 expression is upregulated in endometriosis and contributes to an invasive phenotype. Fertil Steril. 2016; 106(2): 378-385, doi: 10.1016/j.fertnstert.2016.03.032, indexed in Pubmed: 27041028.

7. Manthe RL, Muro S. ICAM-1-Targeted Nanocarriers Attenuate Endothelial Release of Soluble ICAM-1, an Inflammatory Regulator. Bioeng Transl Med. 2017; 2(1): 109-119, doi: 10.1002/btm2.10050, indexed in Pubmed: 28713860

8. Pabalan N, Jarjanazi $\mathrm{H}$, Christofolini DM, et al. Association of the intercellular adhesion molecule-1 (ICAM-1) gene polymorphisms with endometriosis: a systematic review and meta-analysis. Arch Gynecol Obstet. 2015; 292(4): 843-851, doi: 10.1007/s00404-015-3705-2, indexed in Pubmed: 25859827.

9. Palaiologou M, Delladetsima I, Tiniakos D. CD138 (syndecan-1) expression in health and disease. Histol Histopathol. 2014; 29(2): 177-189, doi: 10.14670/HH-29.177, indexed in Pubmed: 24150912.

10. Szatmári T, Ötvös R, Hjerpe A, et al. Syndecan-1 in Cancer: Implications for Cell Signaling, Differentiation, and Prognostication. Dis Markers. 2015 2015: 796052, doi: 10.1155/2015/796052, indexed in Pubmed: 26420915.

11. Amsellem V, Dryden NH, Martinelli R, et al. ICAM-2 regulates vascular permeability and $\mathrm{N}$-cadherin localization through ezrin-radixin-moesin (ERM) proteins and Rac-1 signalling. Cell Commun Signal. 2014; 12: 12, doi: 10.1186/1478-811X-12-12, indexed in Pubmed: 24593809.

12. Chaireti R, LindahITL, Byström B, et al. Inflammatory and endothelial markers during the menstrual cycle. Scand J Clin Lab Invest. 2016; 76(3): 190-194, doi: 10.3109/00365513.2015.1129670, indexed in Pubmed: 26963835.

13. Gorina R, Lyck R, Vestweber $D$, et al. $\beta 2$ integrin-mediated crawling on endothelial ICAM-1 and ICAM-2 is a prerequisite for transcellular neutrophil diapedesis across the inflamed blood-brain barrier. J Immunol. 2014; 192(1): 324-337, doi: 10.4049/jimmunol.1300858, indexed in Pubmed: 24259506.

14. Feduska JM, Aller SG, Garcia PL, et al. ICAM-2 confers a non-metastatic phenotype in neuroblastoma cells by interaction with a-actinin. Oncogene. 2015; 34(12): 1553-1562, doi: 10.1038/onc.2014.87, indexed in Pubmed: 24704826.

15. Hyun YM, Hong CW. Deep insight into neutrophil trafficking in various organs. J Leukoc Biol. 2017; 102(3):617-629, doi: 10.1189/jlb.1RU1216-521R, indexed in Pubmed: 28637894. 
16. Izumi G, Koga K, Takamura $M$, et al. Involvement of immune cells in the pathogenesis of endometriosis. J Obstet Gynaecol Res. 2018; 44(2): 191-198, doi: 10.1111/jog.13559, indexed in Pubmed: 29316073.

17. SoilleuxEJ.DC-SIGN (dendritic cell-specific ICAM-grabbing non-integrin) and DC-SIGN-related (DC-SIGNR): friend or foe? Clin Sci (Lond). 2003; 104(4): 437-446, doi: 10.1042/, indexed in Pubmed: 12653690.

18. Chung YM, Kim BG, Park CS, et al. Increased expression of ICAM-3 is associated with radiation resistance in cervical cancer. Int J Cancer. 2005; 117(2): 194-201, doi: 10.1002/ijc.21180, indexed in Pubmed: 15880373.

19. Kim YG, Kim MiJ, Lim JS, et al. ICAM-3-induced cancer cell proliferation through the PI3K/Akt pathway. Cancer Lett. 2006; 239(1): 103-110, doi: 10.1016/j.canlet.2005.07.023, indexed in Pubmed: 16137825.

20. Park JK, Park SHo, So K, et al. ICAM-3 enhances the migratory and invasive potential of human non-small cell lung cancer cells by inducing MMP-2 and MMP-9 via Akt and CREB. Int J Oncol. 2010; 36(1): 181-192, indexed in Pubmed: 19956847.

21. Schneider C, Kässens N, Greve B, et al. Targeting of syndecan-1 by micro-ribonucleic acid miR-10b modulates invasiveness of endometriotic cells via dysregulation of the proteolytic milieu and interleukin- 6 secretion. Fertil Steril. 2013; 99(3): 871-881.e1, doi: 10.1016/j.fertnstert.2012.10.051, indexed in Pubmed: 23206733.

22. Cicinelli $E$, Trojano $G$, Mastromauro $M$, et al. Higher prevalence of chronic endometritis in women with endometriosis: a possible etiopathogenetic link. Fertil Steril. 2017; 108(2): 289-295.e1, doi: 10.1016/j.fertnstert.2017.05.016, indexed in Pubmed: 28624114.

23. Echtermeyer F, Streit M, Wilcox-Adelman S, et al. Delayed wound repair and impaired angiogenesis in mice lacking syndecan-4. J Clin Invest. 2001; 107(2): R9-RR14, doi: 10.1172/JCI10559, indexed in Pubmed: 11160142. 